\title{
Extraction of Permeability Variations from P-Wave Velocity Dispersion Data
}

\author{
B. Milkereit, W. Pun and B. Harris
}

\section{Summary}

Permeability is a key parameter for the evaluation of subsurface formations in groundwater and hydrocarbon exploration. We utilize broadband full-waveform sonic data to exploit Pride's relationship between P-wave velocity dispersion and permeability for porous, fluid-filled media. Frequency dependent $\mathrm{P}$-wave velocities are extracted from multi-channel sonic data during a two-step process: computation of semblance-based velocity spectra at two or more center frequencies followed by a $2 \mathrm{D}$ cross-correlation of the velocity spectra. A comparison with MRI-derived permeability logs confirm that $\mathrm{P}$-wave velocity dispersion logs can be used to map permeability variations. 


\section{Introduction}

The energy of seismic waves propagating through a fluid-filled porous medium is attenuated by various dissipation mechanisms in which the internal friction $\mathrm{Q}^{-1}$ (where $\mathrm{Q}$ is the quality factor) shows a peak at a certain frequency. See for example the Debye peak model in Figure 1. A common denominator for many proposed attenuation models is that $\mathrm{Q}$ and compressional wave velocity are frequency dependent. However, reliable observational data does not exist for both frequency dependent velocities and frequency dependent attenuation across a broad continuous seismic frequency band.

For fluid-filled porous media, Pride (2005) formulated the relationship between velocity dispersion of compressional waves and permeability. Pride's model of fluid-pressure equilibration and associated wave induced flow establishes a link between permeability and P-wave attenuation and velocity dispersion. The absolute peak value of $\mathrm{Q}^{-1}$ (Fig. 1) is independent of permeability. The critical frequency $f_{c}$ at which attenuation is a maximum (Fig. 1) is directly proportional to formation permeability. At the critical frequency, the largest changes in compressional wave velocity can be expected. Consequently, the critical frequency and the associated maximum change in P-wave velocity are directly proportional to permeability. Thus, velocity dispersion measurements of broadband borehole seismic data could provide information about permeability variations.

Figure 1 Attenuation (blue) and velocity dispersion (red) for fluid-filled porous media. Peak attenuation and frequency dependent velocity occur at resonance frequency fc (Liu et al, 1976).

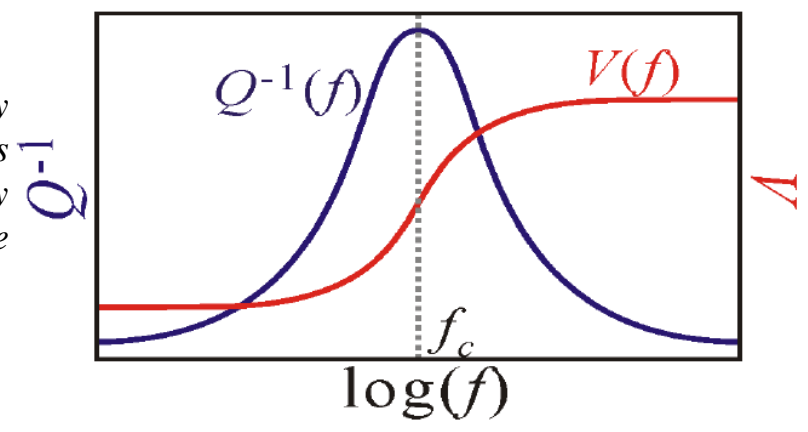

In this project, velocity dispersion in the sonic frequency band $(1 \mathrm{kHz}$ to $30 \mathrm{kHz})$ is investigated. The ideal borehole seismic source based on a continuous chirp does not exist. This leaves two options for the acquisition of suitable full waveform sonic data for velocity dispersion analysis: field measurements with rerun narrow-band sonic logs obtained from seismic sources with different frequency ranges (Pun et al, 2010) or spectral decomposition of multichannel seismic data from a single broadband borehole seismic source (this study).

\section{Method}

Reliable velocity dispersion measurements must be based on an automatic and unbiased processing procedure for analyzing multi-channel full waveform borehole seismic data. The broadband sonic data was obtained from the Mallik gas hydrate production research well project in the Mackenzie Delta of the Canadian Arctic (Dalllimore and Collett, 2005). The sonic data were acquired with a broadband borehole seismic source (Schlumberger DSI (dipole shear sonic imager) operated in monopole mode). In order to perform compressional wave velocity dispersion analysis, the broadband seismic data are decomposed into its low and high frequency components centered at $3 \mathrm{kHz}$ and $13 \mathrm{kHz}$.

The multi-channel seismic data are transformed into semblance-based velocity spectra (Milkereit, 1987). The algorithm works by summing energy at different slopes (velocities) to find the best stacking velocity for coherent seismic energy. Figure 2 shows the semblance analysis of selected lowpass (pass band 1-6 kHz) and high-pass (pass band 7-to13 kHz) data. P-wave velocity dispersion is observed, with the first break P-wave energy shifted to a higher velocity for the high-pass data. 


\section{Near Surface}

Compressional velocity dispersion is determined by the gradient $\Delta \mathrm{V}_{\mathrm{p}} / \Delta \mathrm{f}$. An automatic crosscorrelation method is used to compute the velocity from the semblance-based velocity spectra shown in Figure 2. The coherency values of the two frequencies are cross-correlated to find $\Delta \mathrm{V}_{\mathrm{p}}$ at which the maximum correlation occurs. The correlation can be performed either in 1D (along the velocity axis) or $2 \mathrm{D}$ (along velocity and time) to match the maxima of the velocity spectra.

Figure 3 shows the cross-correlation of the low and high frequency velocity spectra in Figure 2. The zero-lag line indicates no evidence for velocity dispersion. The contour lines of the cross-correlation coefficients indicate a positive velocity lag of approximately $100 \mathrm{~m} / \mathrm{s}$ (high frequency data travel faster than low frequency data). This corresponds to a P-wave velocity dispersion of about $4 \%$.

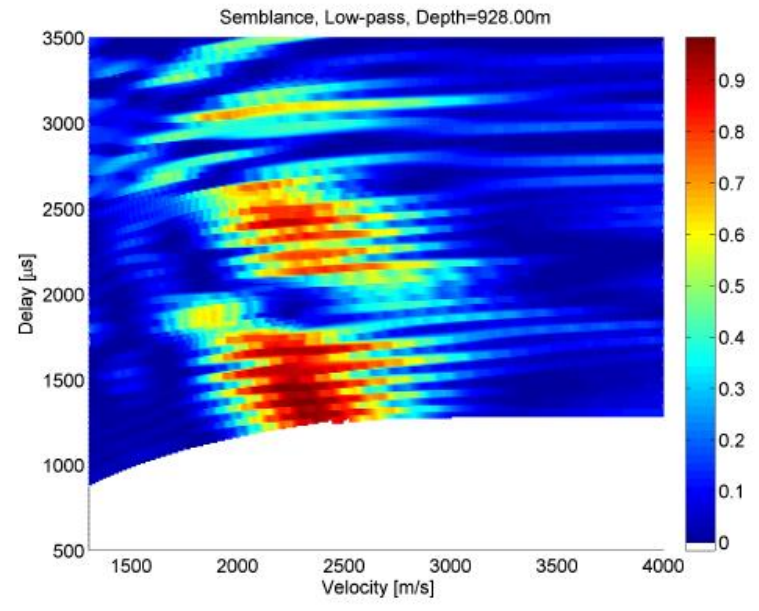

(a) Low-pass, $928.0 \mathrm{~m}$ depth

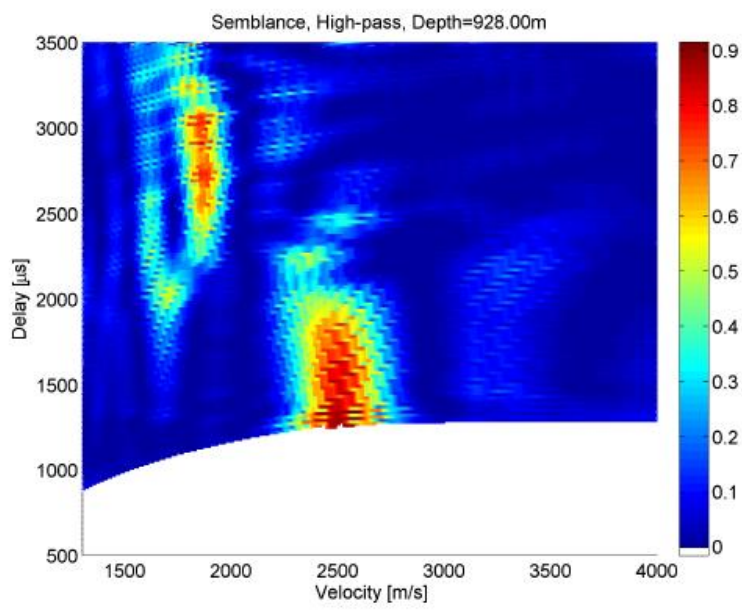

(b) High-pass, $928.0 \mathrm{~m}$ depth

Figure 2 Semblance-based velocity spectra derived from low-pass and high-pass filtered multichannel sonic data.

The velocity dispersion data from individual depth levels along the borehole must be transformed into velocity dispersion logs. An automatic and robust method is the correlation ratio method. The method is closely related to visual inspection procedures. The cross-correlation contour lines define areas with either positive or negative bias. Within the first break window, the sums of the cross-correlation values corresponding to positive and negative velocity lags are computed. The P-velocity dispersion $\log$ is based on the ratio of the two sums (positive versus negative lags). A P-wave velocity dispersion value that lie between 0 and 1 corresponds to negative lags, a value of 1 indicates no evidence for velocity dispersion and a value greater than 1 correspond to positive lags where higher frequencies travel with higher compressional wave velocity. The results of the correlation ratio method are based on a number of cross-correlation values and therefore do not vary greatly with data quality (seismic waveform, number of channels, signal-to-noise ratio, etc.).

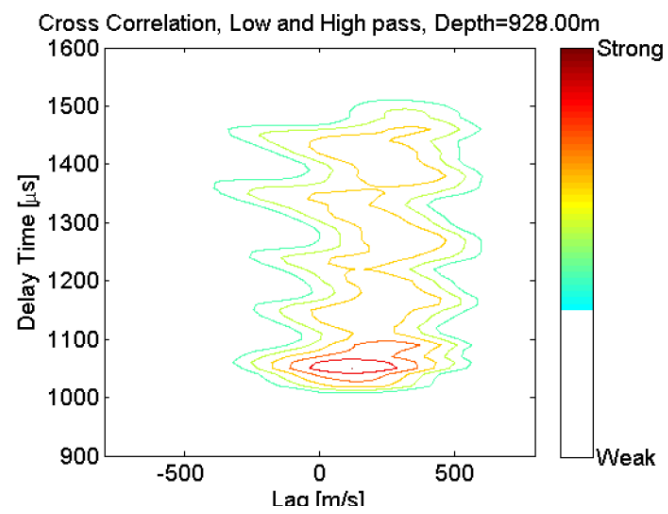

Figure 3 Cross-correlation of semblancebased velocity spectra shown in Fig. 2. Note the positive shift towards higher $P$ wave velocities for the higher frequency data. 


\section{Near Surface}

\section{Field Example}

A wide range of geophysical logs and petrophysical data are available for the Mallik gas hydrate research project (Dallimore and Collett, 2005). Full waveform sonic data, permeability and velocity dispersion logs from the Mallik gas hydrate research well are shown in Figure 4. Depth intervals with early (fast) P-wave arrivals correspond to high gas hydrate saturation. The shallow interval (880$910 \mathrm{~m}$ depth) exhibits elevated $\Delta \mathrm{V}_{\mathrm{p}}$ dispersion data. This correlates well with the MRI-based permeability log (Kleinberg et al., 2005). The gas hydrate-rich interval (910 $\mathrm{m}$ depth and below) shows relatively low $\Delta \mathrm{V}_{\mathrm{p}}$ velocity dispersion and low permeability estimates.

The interval with high permeability and high velocity dispersion (at 901-906 $\mathrm{m}$ depth) is located immediately above a thick interval of massive gas hydrates. This zone may represent a preferred fluid (water) pathway above the massive gas hydrate zone.

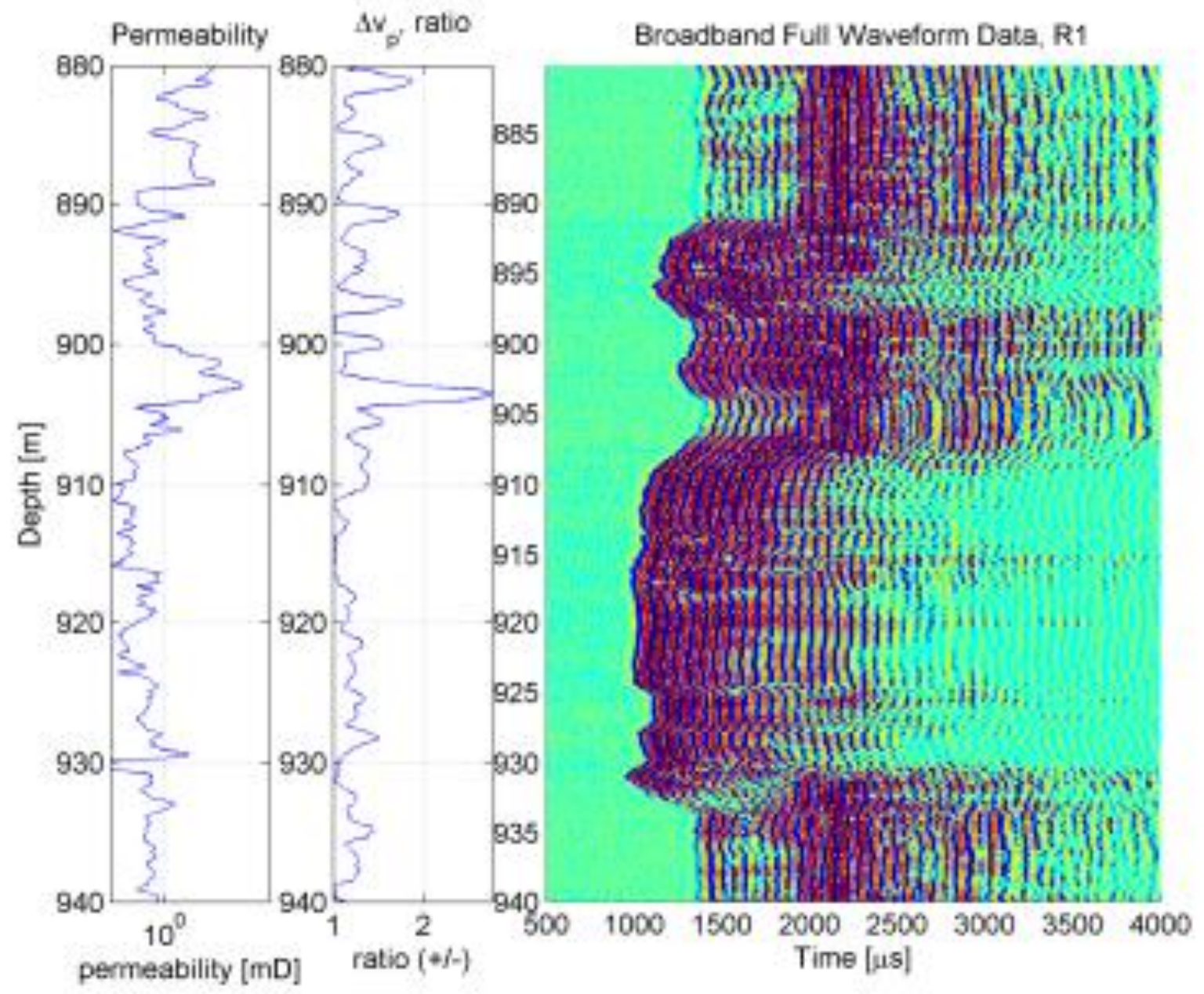

Figure 4 Comparison of the MRI-derived permeability log with P-wave velocity dispersion data. For reference, single channel full waveform sonic data (right panel) highlight the fast gas hydrate formations (early arrivals) and the slow fluid-saturated sedimentary formations (Mallik gas hydrate exploration well, Mackenzie Delta, Canada). The interval between $880 \mathrm{~m}$ and $910 \mathrm{~m}$ depth shows both elevated permeabilities and $P$-wave velocity dispersion data. 


\section{Conclusions}

Pride's (2005) formulation of poroelastic effects on seismic waves provides an elegant mechanism for linking hydrological properties (such as permeability and fluid type) to geophysical parameters (such as frequency dependent velocities and attenuation). P-wave velocity dispersion data was extracted from multi-channel full-waveform sonic data. An automatic processing sequence, based on the crosscorrelation of semblance-based velocity spectra, was developed to compute P-wave velocity dispersion logs. A comparison of MRI-based permeability logs shows that elevated levels of P-wave velocity dispersion correlate well with zones of elevated permeability readings.

Currently there exists no ideal borehole seismic tool for carrying out measurements for P-wave velocity dispersion analysis. The ideal borehole seismic tool would utilize a broadband chirp signal (with a low frequency of a few hundred $\mathrm{Hz}$ and a high frequency of at least $30 \mathrm{kHz}$ ). The wide frequency spectrum of a chirp-based borehole seismic source could then be used to provide continuous measurements of frequency dependent velocities and attenuation (Sun et al., 2009) as required more accurate permeability estimations for groundwater and hydrocarbon reservoirs.

\section{Acknowledgements}

The Mallik full waveform sonic and MRI-based permeability data were acquired as part of the JAPEX/JNOC/GSC et al Mallik 5L-38 gas hydrate research well. This research project was funded by NSERC.

\section{References}

Dallimore, S. R. and Collett, T. S. [2005] Scientific results from the Mallik 2002 gas hydrate production research well program, Mackenzie Delta, Northwest Territories, Canada, Geological Survey of Canada Bulletin 585.

Kleinberg, R.L., Flaum, C., and Collett, T. S. [2005] Magnetic resonance log of JAPEX/JNOC/GSC et al. Mallik 5L-38 gas hydrate production research well: gas hydrate saturation, growth habit and relative permeability, Geological Survey of Canada, Bulletin 585, 10p.

Liu, H-P., Anderson D. L., and Kanamori, H. [1976] Velocity dispersion due to anelasticity; implications for seismology and mantle composition, Geophysical Journal of the Royal Astronomical Society, 47, 41-58.

Milkereit, B. [1987] Decomposition and inversion of seismic data - an instantaneous slowness approach, Geophysical Prospecting, 35, 875-894.

Pride, S.R., [2005] Relations between seismic and hydrological properties, in Y.Rubin, and S.S. Hubbards, eds., Hydrogeophysics: Springer, 253-290.

Pun, W., Milkereit, B., and Harris, B. [2010] Investigation of frequency dependent velocities in fluidfilled porous media, GeoCanada 2010 Conference, Expanded Abstracts, 4p.

Sun, L. F., Milkereit, B. and Schmitt, D. R. [2009] Measuring velocity dispersion and attenuation in the exploration seismic frequency band, Geophysics, 74, 113-122. 